\title{
THE EQUIVALENT SERIES RESISTANCE IN ELECTROLYTIC CAPACITORS
}

\author{
F. G. HAYATEE \\ Electrical Engineering Department, College of Engineering, University of Mosul, Mosul, Iraq
}

(Received October 30, 1973; in final form September 24, 1974)

\begin{abstract}
The analysis of electrolytic capacitors as a distributed network is examined. The parameters contributing to the equivalent series resistance (E.S.R.) have been determined for various production capacitors at low and high frequencies.
\end{abstract}

\section{INTRODUCTION}

Electrolytic capacitors can be represented by an equivalent circuit consisting of a series combination of $R, L$ and $C$ elements. A typical impedencefrequency characteristic in shown in Figure 1. The impedance becomes inductive at frequencies which are for most applications beyond the region of capacitor operations.

The equivalent series resistance $R$ of the capacitor is responsible for heat generation and temperature rise in the capacitor. It also has a damping effect in fast charge/discharge applications. Therefore, it is desirable to reduce the equivalent series resistance (E.S.R.) to a minimum.

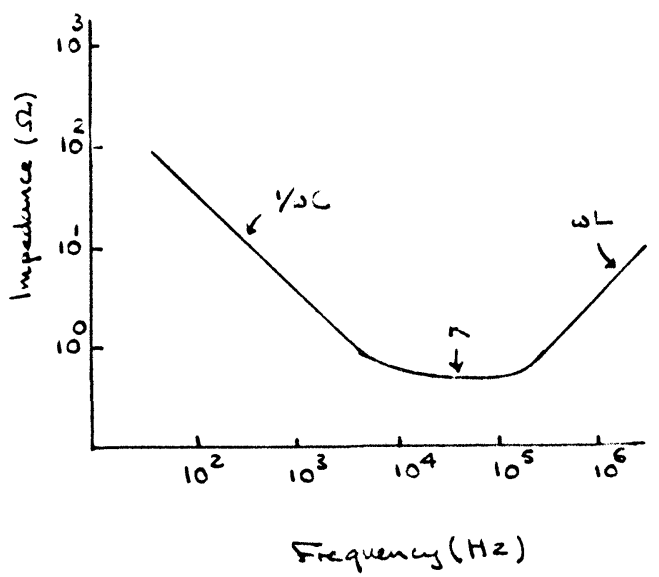

FIGURE 1 Typical impedance-frequency characteristics of an electrolytic capacitor.
The construction and performance of electrolytic capacitors have been reviewed extensively. ${ }^{1-5}$ The most common type of construction consists of an etched aluminium anode and high grain cathode foils separated by electrolyte impregnated low density tissues. The dielectric is provided by the anodization of the anode, and its thickness is determined by the anodization voltage and other anodization parameters, such as temperature and the constituents of the anodization solution. ${ }^{6}$

The total impedance of a capacitor has been evaluated by treating the capacitor as a distributed network. This has been applied to plain foil capacitors $^{\prime}$ and to the evaluation of the impedance of tunnels in etched foils. ${ }^{3}$ More elaborate study has been made of various types of electrolytic capacitors including sintered-anode, etched foil, vacuum deposited and plain foil capacitors. ${ }^{8}$ The use of two transmission line treatment has recently been employed in the analysis of an etched foil electrolytic capacitor. ${ }^{4}$

The exact transmission line model is extremely complex for etched foil capacitors. However, many approximations can be introduced without changing the final results drastically. The following approximations can be made:

1) Inductive effects due to winding and tunnels can be ignored. In practice, inductance is mainly due to lugs and leads. ${ }^{4}$

2) The capacitance of impregnated tissues is insignificant compared with the capacitance of the anode. ${ }^{3}$

3) The capacitance and resistance of the cathode foil can be lumped together with those of the anode 


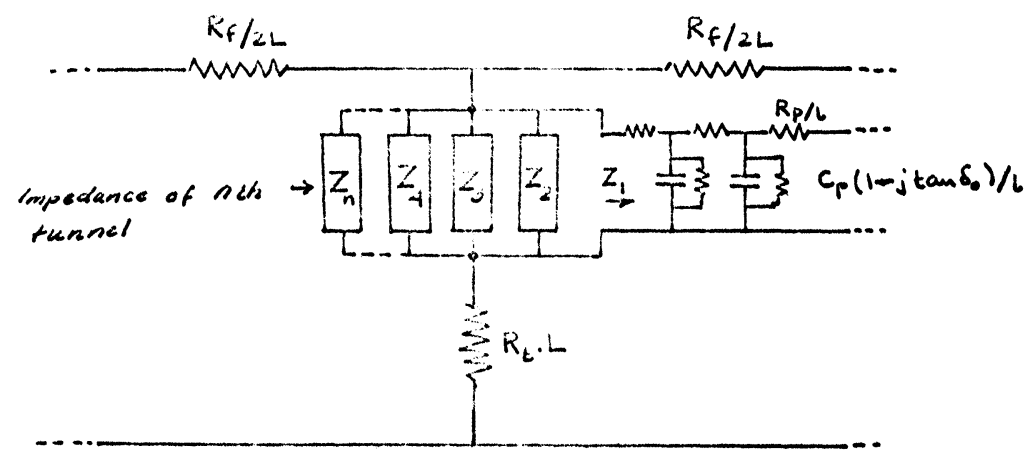

FIGURE 2 Transmission line representation of a unit length of unrolled capacitor.

foil. This is especially true for low voltage capacitors and high gain cathode foil. ${ }^{7}$

4) Interaction between the turns of the wound capacitor can be ignored. The effect of winding is accounted for merely by doubling the admittance of the unrolled plates. This results from the utilization of both sides of each foil when rolled. ${ }^{7}$

With the above simplifications the equivalent circuit of a unit length of an unrolled capacitor can be represented by the circuit shown in Figure 2. The analysis of this circuit shows that below two critical frequencies $f_{\mathrm{c} 1}$ and $f_{\mathrm{c} 2}$ the capacitance $C$ and resistance $R$ of the capacitor are given by: ${ }^{4}$

$$
\begin{gathered}
C=\Sigma C_{p} \\
R=1 / 2 R_{t}+1 / 3 \Sigma R_{p}+1 / 3 R_{f}+R_{o x}+R_{i}
\end{gathered}
$$

where $C_{p}$ is the lumped capacitance of a single tunnel and the summation is for all tunnels on both sides of foil.

$R_{p}$ is the resistance of electrolyte in a tunnel.

$R_{t}$ is the resistance of electrolyte impregnated tissues separating the anode and cathode foils.

$R_{f}$ is the resistance of anode and cathode foils.

$R_{i}$ is the resistance due to lugs and connections.

$R_{o x}$ is the resistance due to dielectric losses.

This is given by:

$$
R_{o x}=\tan \delta_{0} / \omega C
$$

where $\tan \delta_{0}$ is the loss of the oxide.

The two critical frequencies are given by

$$
\begin{aligned}
f_{c 1} & =\frac{1}{2 \pi R_{p} C_{p}} \\
f_{c 2} & =\frac{1}{2 \pi C R_{f}}
\end{aligned}
$$

Above these frequencies the resistance and capacitance of the transmission line begin to fall with frequency according to $f^{-1 / 2}$ law. ${ }^{4}$ The oxide loss is almost frequency independent and thus the resistance $R_{o x}$ is expected to fall linearly with frequency. It is therefore expected that at high frequencies the E.S.R. is mainly due to the resistance of tissues and small contributions from lugs and connections.

\section{EXPERIMENTAL}

A large number of capacitors were made under production conditions. Plain and etched foils were used together with four types of electrolytes and varying anode-cathode separation. A batch of five capacitors was made for each specific type, and the average of each batch was taken.

In all cases the anode plate was formed at $115 \mathrm{~V}$. The capacitance of the plain anode foil was $0.78 \mathrm{mF}$ / $\mathrm{m}^{2}$ while that of the etched anode was $12.5 \mathrm{mF} / \mathrm{m}^{2}$. The cathode was of a low gain type with a capacitance of $0.2 \mathrm{~F} / \mathrm{m}^{2}$.

Measurements of capacitance and $\tan \delta_{0}$ for different frequencies were carried out using standard bridge techniques.

\section{RESULTS AND ANALYSIS}

\subsection{The Critical Frequencies $f_{\mathrm{c} 1}$ and $f_{\mathrm{c} 2}$}

According to theoretical analysis the E.S.R. in a capacitor can be defined by Eq. 2 below the two critical frequencies $f_{\mathrm{c} 1}$ and $f_{\mathrm{c} 2}$. Above those two frequencies the main contribution comes from the impregnated tissue. These frequencies can be evaluated from Eqs. (4) and (5).

The resistance $R_{p}$ and capacitance $C_{p}$ of a tunnel are given by: 


$$
\begin{aligned}
& R_{p}=\rho_{\mathrm{e}} \frac{l}{\pi r^{2}} \\
& C_{p}=\frac{2 \pi \epsilon_{0} \epsilon l}{\ln \frac{r+d}{d}}
\end{aligned}
$$

where $l$ is the length of the tunnel, $r$ is the radius of the formed tunnel, $d$ is the thickness of the oxide, $\rho_{e}$ is the resistivity of the electrolyte, and $\epsilon$ is the dielectric constant of the oxide. Substituting typical values of $l, r, d$ and $\rho_{e}$ yields critical frequency $f_{\mathrm{c} 1}$ of the order of several $\mathrm{kHz}^{4}$

The second critical frequency $f_{\mathrm{c} 2}$ depends on the resistivity of the foil and the total capacitance of the plates. For a capacitor with foil resistivity of $\rho_{f} \Omega /$ square and foil capacitance of $\mathrm{kF} / \mathrm{m}^{2}$, Eq. (5) can be expressed in the form:

$$
f_{c 2}=\frac{1}{2 \pi \rho_{f} k L^{2}}
$$

where $L$ is the length of the plate. In the test capacitors $\rho_{f}$ was found to be $1.6 \times 10^{-3} \Omega /$ square, $k=12.5 \mathrm{~m} F / \mathrm{m}^{2}$ and plate length was $0.25 \mathrm{~m}$. These figures give $f_{\mathrm{c} 2}$ in the region of $150 \mathrm{kHz}$. For most practical cases $f_{\mathrm{c} 2}$ is much lower than this value as the foil capacitance is larger and so is the length of the plates.

It is evident from above that the contributions of electrolyte in tunnels and the foil resistance are present in the region of $100 \mathrm{~Hz}$, while at $150 \mathrm{kHz}$ and above these are expected to become negligible.

\subsection{Contribution of Connections and Foil Resistance}

The contribution of connections (lugs, leads and terminals) to the total resistance is usually negligible, especially in small capacitors. For capacitors with axial leads and with lugs rivetted to the deck and stitched to plates the resistance $R_{i}$ was found to be in the region of 5-10 milliohms.

The resistance of anode and cathode foils were measured using straight forward d.c. technique. The total resistivity of anode and cathode was found to be $1.6 \times 10^{-3} \Omega$ /square (per unit thickness of foil). For plate of size $0.025 \mathrm{~m} \times 0.25 \mathrm{~m}$ the resistance $R_{f}$ is 16 milliohm. In the worst case where foil length is 100 times the width (this gives a capacitor with equal height and diameter) the resistance $R_{f}$ is $0.16 \Omega$. Transmission line analysis shows that only one third of this is contributed to E.S.R.

\subsection{Oxide Losses}

The loss tangent of aluminium oxide is almost frequency independent. Its value depends upon the purity of aluminium and conditions of oxide formation. Mechanical flows and grain boundaries can increase the $\operatorname{loss}^{1}$. For a typical value of $2 \%{ }^{4}$ the resistance due to oxide is

$$
R_{o x} \equiv \frac{\tan \delta_{0}}{\omega C}=\frac{0.02}{\omega C} \mathrm{ohm} .
$$

In small capacitors, e.g. $1 \mu F$, the series resistance due to oxide is approximately $32 \Omega$ at $100 \mathrm{~Hz}$. This represents the main contribution to E.S.R.

The capacitors investigated had an average capacitance of $4.85 \pm 1 \% \mu F$ with plain anode and $76.0 \pm 4 \% \mu F$ with etched anode. Consequently, their oxide losses are $6.6 \pm 0.07 \Omega$ and $0.42 \pm .02 \Omega$ at $100 \mathrm{~Hz}$ respectively. At $100 \mathrm{kHz}$ these drop by a factor of 1000 , thus becoming negligible.

\subsection{Contribution of Impregnated Tissues}

The resistance of impregnated tissue with resistivity $\rho_{t}$ is given by the relationship:

$$
R_{t}=\rho_{t} \frac{t}{A}
$$

where $t$ is the thickness of tissues and $A$ is the area of the capacitor plate.

According to analysis, the resistance E.S.R. at high frequencies is predicted to be mainly due to tissues. The experimental vertification of this requires measurements of high frequency resistance for different electrolytes and tissue thickness. The electrolytes shown in Table I were used.

Results in Figure 3 shows the dependence of the high resistance on tissue thickness for several electrolytes. The frequency of measurements were chosen where the impedance was minimum (Figure 1). In all cases this frequency was above $100 \mathrm{kHz}$, which was well above the critical frequencies.

TABLE I

Electrolytes

\begin{tabular}{lc}
\hline Type & $\begin{array}{c}\text { Nominal } \rho_{e} \text { at } 50 \mathrm{kHz}\left(20^{\circ} \mathrm{C}\right) \\
(\Omega / \mathrm{m})\end{array}$ \\
\hline E 92 & 1.2 \\
E 93 & 18.0 \\
E 94 & 31.0 \\
E 98 & 7.7 \\
\hline
\end{tabular}


- plin arest

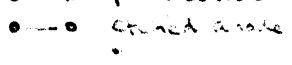

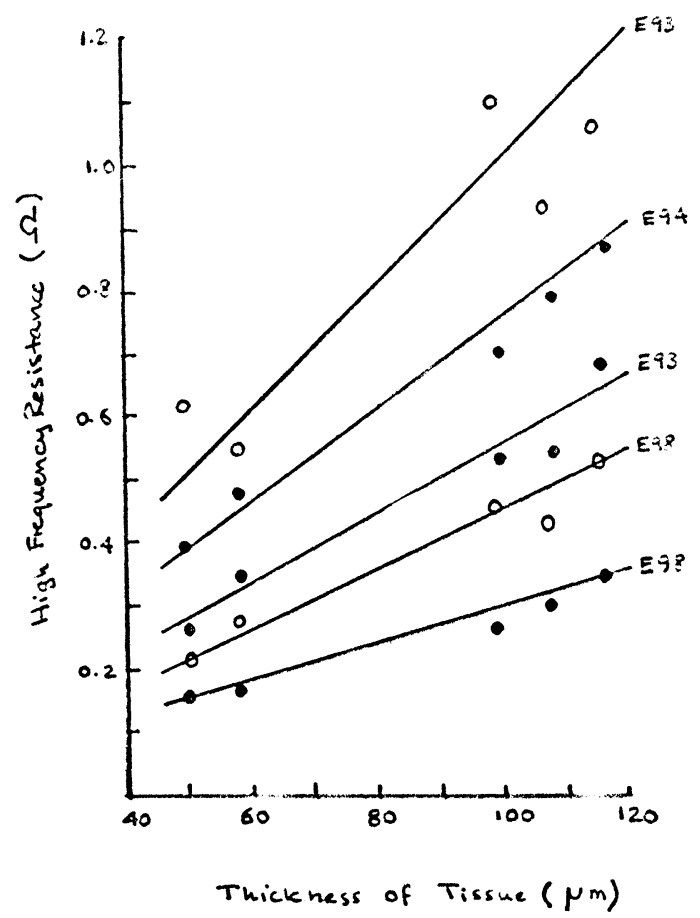

FIGURE 3 Dependence of high frequency resistance on tissue thickness.

The differences between the tissue resistances in etched foil and plain foil capacitors for the same electrolyte cannot be attributed to any one cause with certainty. It could be the result of variation in the resistivity of one type of electrolyte at different impregnation cycles. It could also be due to different winding tension in the capacitors, and the timetemperature history during and after impregnation. ${ }^{9}$

The results in Figure 3 clearly indicate the direct proportionality between resistance and tissue thickness. This suggests that the high frequency resistance is due to impregnated tissue. Further evidence of this

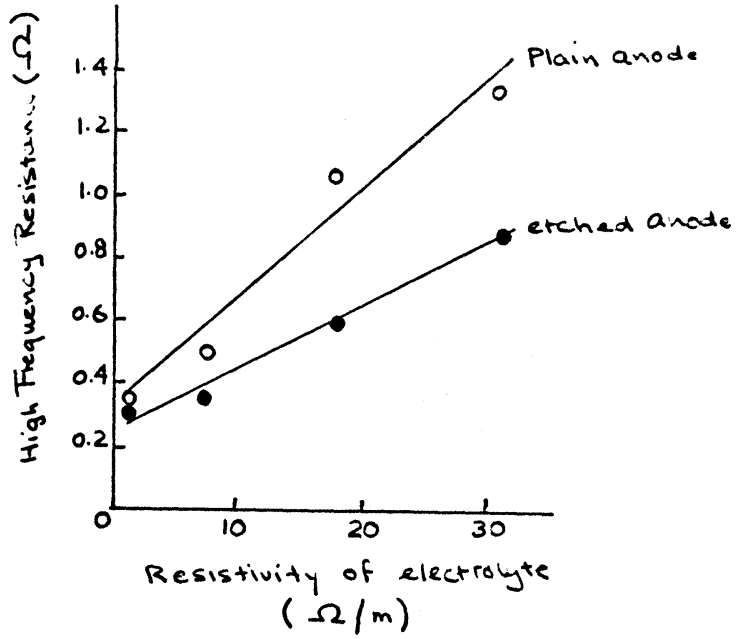

FIGURE 4 Dependence of high frequency resistance on electrolyte resistivity.

is the dependence of the high frequency resistance on electrolyte resistivity (Figure 4).

\subsection{Contribution of Tunnels}

Since it is expected that at high frequencies the resistances of oxide, foil and tunnels become negligible it is possible to estimate the contribution of tunnels from high frequency data $R_{h f}$ and those at $100 \mathrm{~Hz}$. The tunnel resistance can be evaluated from:

$$
\Sigma R_{p}=3\left[R_{(100 \mathrm{~Hz})}-R_{o x(100 \mathrm{~Hz})}-R_{h f}\right]
$$

The term $R_{o x}(100 \mathrm{~Hz})$ due to dielectric was calculated using Eq. (9) and assuming a dielectric loss of $2 \%$. The contribution of tunnels is expected to be independent of tissue thickness and dependent of electrolyte resistivity. The results in Table II show the calculated values of $\Sigma R_{p}$ for various electrolytes and tissue thickness. These results clearly indicate their independence of tissue thickness. Moreover, the plot of tunnel contribution versus electrolyte resistivity for $120 \mathrm{~m}$ tissue thickness, Figure 5, shows direct proportionality between the two to within $\pm 10 \%$.

TABLE II

\begin{tabular}{lclllll}
\hline & \multicolumn{6}{l}{ Tunnel contribution for tissue thickness of } \\
\cline { 3 - 6 } Electrolyte & $\begin{array}{c}\text { Nominal } \\
\text { resistivity }\end{array}$ & $120 \mu \mathrm{m}$ & $105 \mu \mathrm{m}$ & $90 \mu \mathrm{m}$ & $60 \mu \mathrm{m}$ & $45 \mu \mathrm{m}$ \\
\hline E 93 & 18.0 & 2.4 & 1.9 & 2.5 & 2.2 & 2.0 \\
E98 & 7.7 & 1.3 & 1.2 & 1.1 & 1.2 & 1.0 \\
E94 & 31.0 & 2.8 & 2.5 & 2.8 & 2.8 & 2.2 \\
E92 & 1.2 & 1.0 & - & - & - & - \\
\hline
\end{tabular}


- Plam cinodic

o...o Etched anods

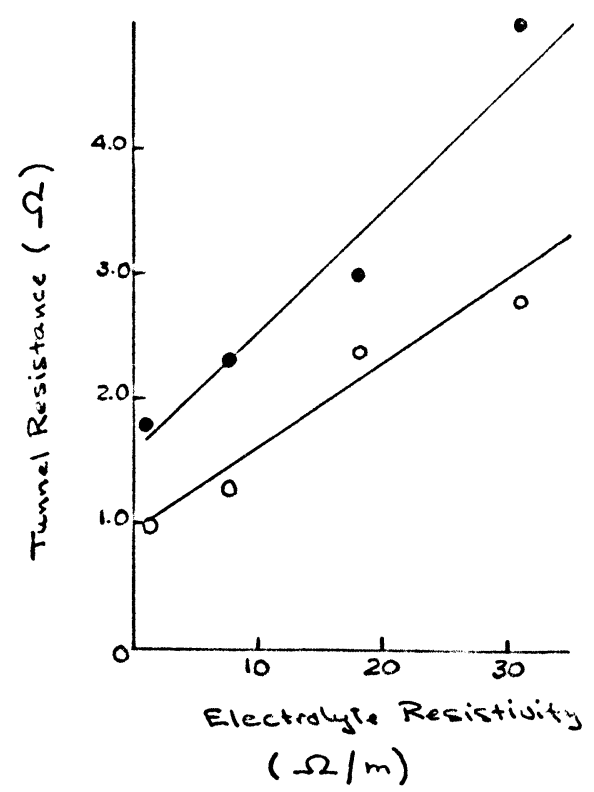

FIGURE 5 Dependence of tunnel contribution on resistivity of electrolyte.

In the case of the plain anode foil, there are larger fluctuations in the values of the calculated tunnel contribution (Table III). Nevertheless, they still seem to be independent of the tissue thickness. It is puzzling however, to find that these contributions are larger than those found in etched foil.

It is very hard to give a definite explanation for this discrepancy. This can arise from variation in the dielectric loss from one foil to another. For example, a value of $2.1 \%$ for $\tan \delta_{o}$ instead of $2 \%$ nearly halves the contributions given in Table III, while those in Table II will be reduced by less than $3 \%$. This can also be the cause of the larger fluctuations noted in Table III.

\subsection{Dependence of Capacitor Resistance on Plate Area and Type of Electrolyte}

Five batches of $100 \mathrm{~V}$ capacitors were produced with different plate areas, but using fixed tissue thickness and two types of electrolytes E 93 and E 98 .

The resistance of the capacitors was determined at $100 \mathrm{~Hz}$ and the contribution of oxide, lugs and foil were subtracted. The remainder was plotted as a function of the reciprocal of plate area, Figure 6.

The results show a straight line relationship. The ratio of the slopes of the lines for E 93 and E 98 is 1.85. This is close to the ratio of the resistivities of the two electrolytes at $100 \mathrm{~Hz}$, which was found to be nearly 2 . These findings indicate that the calculated resistances are due to electrolyte in tunnels and impregnated tissues.

\subsection{Estimation of Tunnel Density and Length on Etched Anode Foil}

The density and size of tunnels depend on the process of etching and the microstructure of the foil. ${ }^{5}$ The

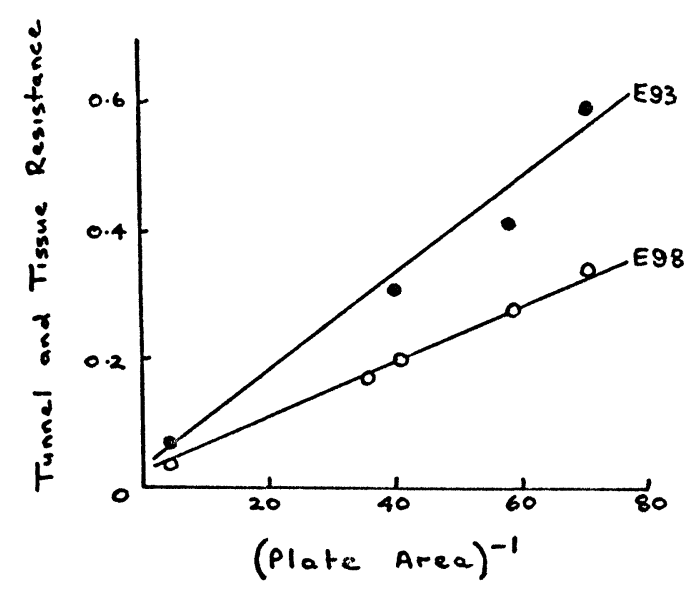

FIGURE 6 Dependence of tunnel and tissue resistance on the reciprocal of plate area.

TABLE III

\begin{tabular}{lclllll}
\hline & & \multicolumn{5}{l}{ Tunnel contribution for tissues thickness of } \\
\cline { 3 - 6 } Electrolyte & $\begin{array}{c}\text { Nominal } \\
\text { resistivity }\end{array}$ & $120 \mu \mathrm{m}$ & $105 \mu \mathrm{m}$ & $90 \mu \mathrm{m}$ & $60 \mu \mathrm{m}$ & $45 \mu \mathrm{m}$ \\
\hline E 93 & 18.0 & 3.0 & 3.1 & 4.0 & 3.1 & 5.3 \\
E 98 & 7.7 & 2.3 & 2.1 & 3.3 & 2.1 & 4.0 \\
E 94 & 31.0 & 5.0 & - & - & - & - \\
E 92 & 1.2 & 1.8 & - & - & - & - \\
\hline
\end{tabular}


tunnel has a Gaussian distribution in length and diameter. $^{4}$

The average tunnel diameter in the anode used was $3.5 \times 10^{-7} \mathrm{~m}$ at a formation voltage of $20 \mathrm{~V}$. At $115 \mathrm{~V}$ the diameter of the tunnel and thickness of the dielectric can be evaluated. ${ }^{4}$ These are $1.8 \times 10^{-7} \mathrm{~m}$ and $1.6 \times 10^{-7} \mathrm{~m}$ respectively.

The cathode is of the same type used by Morley and Campbell ${ }^{4}$. The resistance of electrolyte in the cathode tunnels is determined for electrolyte E 93 and found to be nearly $0.2 \Omega$ in a capacitor. This is negligible compared to the total resistance due to anode and cathode tunnels (Table II).

The total anode capacitance and tunnel resistance are known experimentally. These are used in Eqs. (6) and (7) to determine tunnel length and density. The average tunnel length is found to be $25 \times 10^{-6} \mathrm{~m}$ and the density is $1.4 \times 10^{12}$ tunnels $/ \mathrm{m}^{2}$. These are close to the values normally found ${ }^{4,5}$

\section{CONCLUSIONS}

The transmission line analysis of electrolytic capacitors has been examined experimentally and the results indicate close agreement with theoretical predictions. The high frequency resistance is shown to be mostly due to impregnated tissue. The low frequency resistance, on the other hand, is the sum of the contributions of all parameters. The relative contribution of each parameter can be evaluated for any capacitor and for small capacitors it is found that resistance is mostly due to the dielectric loss.

\section{ACKNOWLEDGEMENTS}

The author is grateful to Professor Campbell, Dr. P. J. Harrop and Dr. A. R. Morley for helpful discussions. I am also grateful to the Plessey Co. Ltd., for permission to publish this paper.

\section{REFERENCES}

1. F. J. Burger and L. Young, Prog. Dielectrics 51 (1963).

2. D. S. Campbell, Radio Electron. Eng. 41 (1) 5 (1971).

3. R. H. Broadbent, J. Electrochem. Soc. 6 (5) 163 (1968).

4. A. R. Morley and D. S. Campbell, Radio Electron. Eng. 43 421 (1973).

5. C. G. Dunn, R. B. Bolon, A. S. Alwan and A. W. Stirling, J. Electrochem. Soc. 18 (2) 381 (1971).

6. L. Young, Anodic Oxide Films (Academic Press, New York, 1961).

7. R. M. Peekema and J. P. Beesley, J. Electrochem. Soc. 6 (5) 166 (1968).

8. K. Nishitani, Electr. Eng. Jap. 89 (7) 1 (1969).

9. R. S. Alwitt, J. Electrochem. Soc. 116 (7) 1024 (1969). 

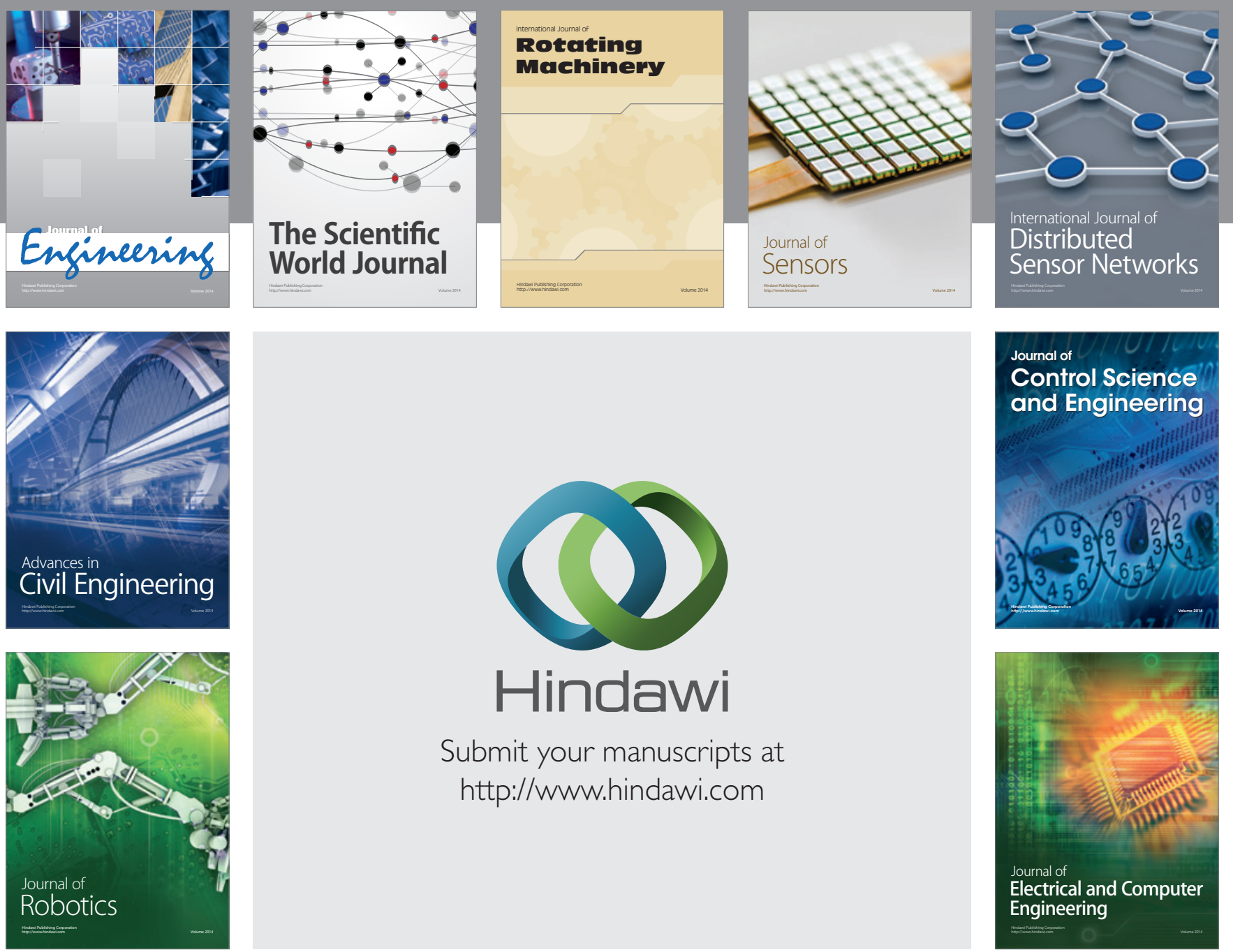

Submit your manuscripts at

http://www.hindawi.com
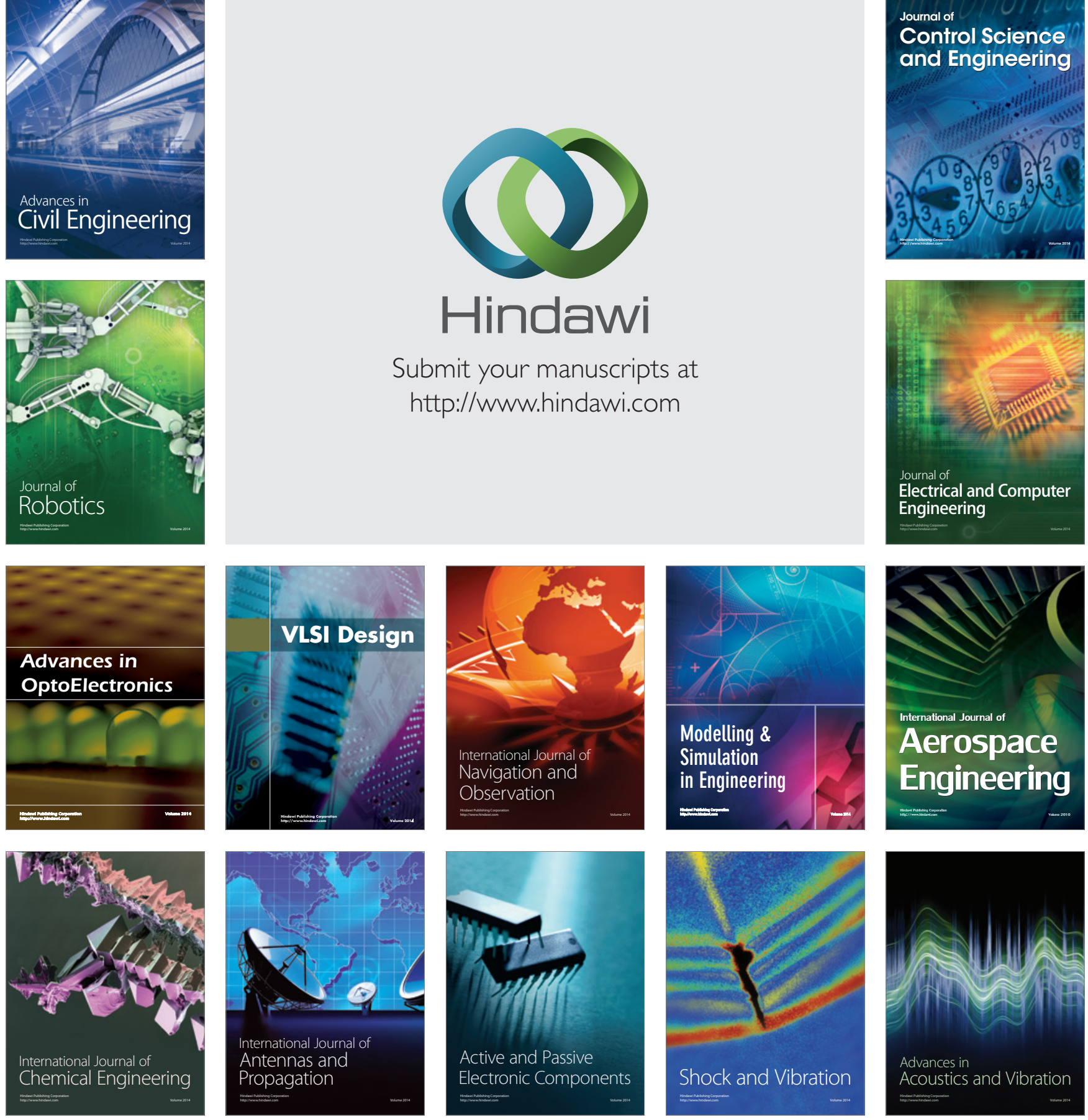